\title{
ESTABILIDAD Y CONFIABILIDAD DE LOS NUEVOS HÍBRIDOS DE MAÍZ EN COMPARACIÓN AL TESTIGO REGIONAL HB-83, 1998-20001
}

\author{
Ismael Camargo ${ }^{2}$, Román Gordón ${ }^{2}$, Mario Fuentes ${ }^{3}$
}

\begin{abstract}
RESUMEN
Estabilidad y confiabilidad de los nuevos híbridos de maíz en comparación al testigo regional HB-83, 1998-2000. Se realizó un estudio en donde se estimó la estabilidad (AMMI) y la confiabilidad o respuesta normalizada (RNI) de híbridos de grano blanco, evaluados en el ensayo regional de maíz del PCCMCA entre los años 1998 y 2000. En este período se evaluaron 100 genotipos en 48 ambientes de nueve países de Centro América y el Caribe. El estudio permitió identificar genotipos que combinan baja interacción con el ambiente y una respuesta normalizada variando de buena a promedio $(0,6 \geq$ RNI < 0,9) con respecto al testigo. Se determinó la estabilidad y el grado de confiabilidad de la respuesta con respecto al testigo regional HB-83 para cada año. Adicionalmente se realizó el mismo análisis para el combinado de los 12 híbridos coincidentes al menos en dos años del combinado. El análisis combinado indicó que el $25 \%$ de los genotipos presentaron un RNI = 0,8, siendo ellos: CM 933-133, XM 7706 y XM 7708. Seis de los cultivares (el 50\%) presentaron RNI promedio, sobresaliendo el H-59 y CB-HS-9 con valores de 0,73 y 0,75 respectivamente; los otros $25 \%$ mostraron RNI bajo, o sea inferior a 0,6 . El análisis AMMI por su parte identificó al grupo de conformados por el HB-83, CB-HS-11, HN-12 y Trop E 103 como los cultivares con menor interacción con el ambiente. Ambos análisis permitieron verificar que el HB-83 continúa siendo un testigo regional vigente y competitivo por su alta producción y buena estabilidad a través de ambientes contrastantes.
\end{abstract}

\begin{abstract}
Stability and reliability of the new corn hybrids in comparison to the regional control HB-83, 1998-2000. A study was conducted to estimate the stability (AMMI) and the confidence or normalized response (RNI) for a white corn hybrid, assessed in a regional PCCMCA corn trial from 1998 to 2000. In this period, 100 genotypes were evaluated at 48 environments at nine Central America and the Caribbean countries. The RN1 values represents the specific genotype's probability increase over the mean yield, specified by a control. The study allowed to identify genotypes with low environment interaction and of normalized response varying from good to average $(0.6 \geq \mathrm{RNI}<0.9)$ according with the control. The stability and the confidence degree, regarding to the regional control HB-83, was determined every year. In addition, the same analysis was performed with 12 genotypes in common at least in two year of this phase. The combined analysis indicated that $25 \%$ of genotypes showed a RNI $=0.8$, these genotypes were CM 933-133, XM 7706 and XM 7708. Six of them (50\%) showed standard RNI, outstanding the H-59 and CB-HS-9 with RNI values of 0.73 and 0.75 , respectively; the other $25 \%$ presented low RNI, less than 0.6. The HB-83, CB-HS-11, HN12 and Trop E 103 were identified as hybrids with low genotype-environment interaction by the AMMI analysis. Both analysis allowed to verify that HB-83 is still a competitive and current regional control due to its high production and good stability throughout contrasting environments.
\end{abstract}

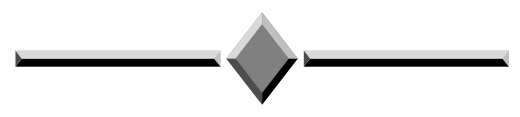

\section{INTRODUCCIÓN}

El ensayo regional de maíz del PCCMCA se inició en 1956, como un instrumento colaborativo para la eva- luación sistemática de cultivares desarrollados por los Programas Nacionales y las Empresas Privadas que operan en la región. La experiencia acumulada ha permitido fortalecer las técnicas de campo para la realización

1 Recibido para publicación en abril del 2002. Presentado en la XLVIII Reunión Anual del PCCMCA. República Dominicana, 2002.

2 Programa de Maíz, Instituto de Investigación Agropecuaria de Panamá (IDIAP). Apdo. 6-4391, El Dorado, Panamá 6A, Panamá. Tel (507) 9933253. E-mail: icamargo@cwpanama.net; rgordonm@cwp.net.p.

4 Programa de Maíz, Instituto de Ciencia y Tecnología Agrícola (ICTA), Apdo. 231-A, Guatemala. E-mail: mrfuentesl@hotmail.com 
de los experimentos, así como el análisis e interpretación de los resultados mediante el uso de metodologías apropiadas para lograr conclusiones acertadas (Córdova et al. 1993).

La interacción genotipo-ambiente merece gran importancia en la evaluación de genotipos desarrollados para diferentes condiciones de producción. Es necesario integrar los conceptos de estabilidad para caracterizar el comportamiento de los cultivares evaluados a través de ambientes contrastantes (Lin et al. 1986, Lin y Binns 1994). La caracterización de la estabilidad de los genotipos evaluados en diferentes sitios y años se ha logrado por medio de los modelos de regresión propuestos por Finlay y Wilkinson (1963), Eberhart y Russell (1966) y, últimamente, el modelo multiplicativo AMMI (Zobel et al. 1988).

El mejoramiento genético es un proceso dinámico que permite evaluar el rendimiento de una serie de cultivares, los cuales generalmente varían de un año a otro, debido a que nuevos cultivares son incluidos para su evaluación mientras otros son eliminados por su pobre comportamiento. La sustitución de cultivares en ensayos regionales repetidos anualmente en ambientes contrastantes, resulta en una serie de datos desbalanceados que requieren análisis especiales para aprovechar la información generada a través de varios años. Algunos autores entre ellos, Hill y Rosenberger (1985), Jones (1988), Eskridge y Mumm (1992), Nuland y Eskridge (1992), Eskridge et al (1993), Jones (1988), Lin y Binns (1994), Eskridge (1997), han propuestos metodologías estadísticas que permiten identificar genotipos superiores utilizando datos desbalanceados.

La metodología propuesta por Jones (1988), permite la comparación de los cultivares contra el testigo utilizando los datos de varios años. Eskridge y Mumm (1992), Eskridge et al. (1993) Nuland y Eskridge (1992), Eskridge (1997), perfeccionaron el análisis de confiabilidad o respuesta normalizada, basados en las diferencias de rendimiento de los cultivares respecto a un testigo adaptado a la región, Lin y Binns (1994) definieron como medida para caracterizar el desempeño genotípico, el cuadrado medio de la distancia entre la respuesta media del cultivar y la respuesta media superior para todos los ambientes, así, el cuadrado medio menor indicará una superioridad del cultivar.

El concepto de confiabilidad propuesto por Eskridge y Mumm (1992), Eskridge et al. (1993) Eskridge (1997), es similar, en principio, al análisis de superioridad propuesto por Lin y Binns (1994). Baker (1996), Indica que ambos conceptos estadísticos la medida de superioridad y el análisis de confiabilidad, representan avances importantes en el entendimiento de los proble- mas del mejoramiento genético, producto de la interacción genotipo-ambiente. Por otro lado, Córdova et al. (1993), Hodges et al. (1995), Camargo et al. (2001), han demostrado la aplicación práctica de esta metodología para determinar la confiabilidad de las ganancias en rendimiento sobre un testigo, e identificar potenciales cultivares para su liberación comercial y para ser utilizados como nuevo testigo para discriminar los cultivares en años subsiguientes. Córdova et al. (1993), basado en sus resultados recomendó el uso del híbrido HB-83 como testigo regional para el ensayo del PCCMCA.

El objetivo del presente estudio fue determinar la estabilidad y confiabilidad para el rendimiento de grano de los nuevos híbridos de maíz de grano blanco con respecto al testigo regional HB-83, a través de ambientes y años contrastantes, así como, verificar la utilidad práctica de estas herramientas en las decisiones asociadas con el cambio tecnológico, que representa la recomendación de un nuevo cultivar.

\section{MATERIALES Y MÉTODOS}

\section{Origen de la información experimental}

Los datos experimentales utilizados en este estudio, provienen de resultados obtenidos de los ensayos colaborativos del PCCMCA de 1998 al 2000. La metodología experimental, como el número de cultivares y localidades fueron descritas por Fuentes y Quemé (1999, 2000 y 2001). Durante este período fueron evaluados en 48 ambientes de nueve países de la región, 102 híbridos de maíz de grano blanco correspondiendo, 76 de las empresas privadas y 26 de los programas nacionales, apoyados por el PRM-CIMMYT.

En este estudio se estimó la puntuación AMMI para caracterizar la estabilidad y la Respuesta Normalizada (Confiabilidad) con respecto al HB-83. Se utilizaron trece híbridos, con dos y tres años de evaluación, fluctuando el número de localidades entre 32 y 48, para los diferentes genotipos (Cuadro 1).

\section{Concepto de respuesta normalizada (RNI) o confia- bilidad de la respuesta}

Según Eskridge et al. (1993), la confiabilidad estadística de la respuesta en rendimiento del i-écimo genotipo se determina a partir de los diferenciales de rendimiento $\left(\mathrm{d}_{\mathrm{i}}\right)$ con respecto al testigo, o sea:

$$
\mathrm{d}_{\mathrm{i}}=\mathrm{P}\left(\mathrm{Y}_{\mathrm{i}}-\mathrm{Y}_{\mathrm{c}}>\mathrm{d}_{\mathrm{i}}\right)
$$


donde:

$\mathrm{P}($.) representa la probabilidad

$\mathrm{Y}_{\mathrm{i}}$ respuesta del híbrido evaluado

$\mathrm{Y}_{\mathrm{c}}$ respuesta del testigo, $\mathrm{y}$

$d_{i}$ es la diferencia entre ambos.

Córdova et al. (1993) indican que pueden ser planteadas las hipótesis alternas en función, a sí el valor promedio de $\mathrm{d}_{\mathrm{i}}$ es mayor o menor que cero es decir:

$$
\begin{aligned}
& \text { Ho: } d_{i}-c \leq 0 \\
& \text { Ha: } d_{i}-c>0
\end{aligned}
$$

Cuadro 1. Listados de los híbridos considerados en el estudio combinado de tres años, institución, los años de evaluación, el número de localidades, y el país de origen. PCCMCA 1998-2000

\begin{tabular}{llccl}
\hline \multicolumn{1}{c}{ Híbridos } & $\begin{array}{c}\text { Institución/ } \\
\text { Empresas }\end{array}$ & $\begin{array}{c}\text { Años } \\
\text { Eval. }\end{array}$ & $\begin{array}{c}\text { Loc's } \\
\text { Eval. }\end{array}$ & \multicolumn{1}{c}{ País } \\
\hline CB-HS-11 & Cristiani B. & 2 & 35 & Guatemala \\
CB-HS-9 & Cristiani B. & 2 & 35 & Guatemala \\
Trop E-101 & S. del Trop. & 2 & 32 & Guatemala \\
Trop E 103 & S. del Trop. & 3 & 48 & Guatemala \\
CMS933133 & CIMMYT & 3 & 48 & México \\
H-59 & CENTA & 3 & 48 & El Salvador \\
HN-12 & INTA & 2 & 32 & Nicaragua \\
VN30F94 & Pioneer & 3 & 48 & México \\
XM-7706 & Asgrow & 2 & 35 & México \\
XM-7708 & Asgrow & 2 & 35 & México \\
HR-93 & Seminal & 3 & 48 & Guatemala \\
HR-99 & Seminal & 2 & 35 & Guatemala \\
HB-831 & ICTA & 3 & 48 & Guatemala \\
\hline
\end{tabular}

1 Testigo regional

Para probar estas hipótesis calculamos primero los valores promedios de $\mathrm{d}_{\mathrm{i}}$ y la desviación estándar de las diferencias a través de todas las localidades $\left(\mathrm{Sd}_{\mathrm{i}}\right)$. Posteriormente se determina el valor estandarizado $\left(\mathrm{d}_{\mathrm{i}} / \mathrm{Sd}_{\mathrm{i}}\right)$ al cual se le determina la Probabilidad Normal Estandarizada (usando la tabla de $\mathrm{Z}$ normal de una cola). A esta probabilidad se le denomina Confiabilidad Normalizada con respecto al testigo $\left(\mathrm{RN}_{\mathrm{i}}\right)$. El análisis de confiabilidad fue calculado para todas las comparaciones de interés, luego se procedió a ordenar de manera descendente los genotipos en función de esta probabilidad.

El valor de $\mathrm{RN}_{\mathrm{i}}$ para cada genotipo representa la probabilidad de que un genotipo específico supere en promedio el nivel de producción establecido por el testigo, en el conjunto de todos los ambientes y años donde se efectuaron las evaluaciones (Eskridge y Mumm 1992, Eskridge et al. 1993 y Eskridge 1997). De acuerdo a Córdova et al. (1993), los genotipos pueden ser clasificados en cuatro categorías según el $\mathrm{RN}_{\mathrm{i}}$ estimado, a saber: a) confiabilidad superior $\left(0,9 \geq \mathrm{RN}_{\mathrm{i}}<1,0\right)$; b) buena $\left(0,8 \geq \mathrm{RN}_{\mathrm{i}}<0,9\right)$; c) promedio $\left(0,6 \geq \mathrm{RN}_{\mathrm{i}}<\right.$ $0,8)$, y; d) baja $\left(\mathrm{RN}_{\mathrm{i}}<0,6\right)$.

\section{RESULTADOS Y DISCUSIÓN}

\section{Estabilidad y confiabilidad respecto al testigo}

La ventaja práctica del análisis de confiabilidad basado en las diferencias de rendimiento de los genotipos respecto al testigo, es que con éste se permite comparar los híbridos en una amplia gama de ambientes y además se determina la estabilidad relativa de los genotipos evaluados sin considerar el conjunto original de materiales, facilitando un mayor aprovechamiento de la información generada a través de varios años en la toma de decisiones acertadas al recomendar la nueva tecnología (híbridos) al productor.

En el Cuadro 2, se presenta un resumen que contiene para cada año de evaluación el número de híbridos, localidades y países involucrados, además, la media general y el rango obtenidos para rendimiento de grano durante el periodo cubierto por el estudio.

El análisis de varianza de acuerdo al modelo AMMI, Cuadro 3, muestra que en los tres años cubiertos por el estudio, la varianza ambiental fue mayor que la varianza de los genotipos, estos resultados eran esperados debido a la gran variabilidad ambiental existente en el mega ambiente Mesoamericano (Fuentes y Quemé 1999, 2000 y 2001). Se observa además, que los tres años fueron bien contrastantes dada las magnitudes de sus varianzas, la mayor variación ambiental se estimó en 1998 y la menor en el año 2000.

Cuadro 2. Años de evaluación, número de genotipos, ambientes, países, medias y rangos de rendimiento de grano.

\begin{tabular}{|c|c|c|c|c|c|}
\hline \multirow[t]{2}{*}{ Años } & \multirow[t]{2}{*}{ Geno. } & \multirow[t]{2}{*}{ Amb. } & \multirow[t]{2}{*}{ Países } & \multicolumn{2}{|c|}{ Rendimiento (t/ha) } \\
\hline & & & & Media & Rango \\
\hline 1998 & 42 & 13 & 8 & 6,07 & $7,10-4,98$ \\
\hline 1999 & 30 & 19 & 8 & 6,52 & $7,47-5,18$ \\
\hline 2000 & 30 & 16 & 9 & 5,81 & $6,70-4,09$ \\
\hline Total & 102 & 48 & & & \\
\hline
\end{tabular}
PCCMCA 1998-2000. 
Cuadro 3. Análisis AMMI para la variable rendimiento de grano (t/ha) de la evaluación de híbridos blancos del PCCMCA. 1998, 1999 y 2000.

\begin{tabular}{lrrrrrr}
\hline Fuente de & \multicolumn{3}{c}{ Grados libertad } & \multicolumn{3}{c}{ Cuadrados Medios** } \\
\multicolumn{1}{c}{ Var. } & $\mathbf{1 9 9 8}$ & $\mathbf{1 9 9 9}$ & $\mathbf{2 0 0 0}$ & $\mathbf{1 9 9 8}$ & $\mathbf{1 9 9 9}$ & $\mathbf{2 0 0 0}$ \\
\hline \multirow{2}{*}{ AMB'S } & 12 & 18 & 15 & 329,9 & 258,5 & 161,4 \\
GEN'S & 41 & 29 & 29 & 13,09 & 15,94 & 13,06 \\
G x A & 492 & 522 & 435 & 1,025 & 1,25 & 1,14 \\
PCA $_{1}$ & 52 & 46 & 43 & 2,155 & 3,27 & 2,97 \\
PCA $_{2}$ & 50 & 44 & 41 & 1,958 & 2,73 & 2,28 \\
Residuo & 390 & 432 & 351 & 0,754 & 0,89 & 0,79 \\
Error & 1066 & 1102 & 928 & 0,586 & 0,49 & 0,46 \\
Total & 1637 & 1709 & 1439 & 3,488 & 3,75 & 2,65 \\
\hline
\end{tabular}

**Todas las fuentes de variación presentaron diferencias altamente significativas $(\mathrm{P}<0,01)$ en 1998, 1999 y 2000.

Por otro lado, la varianza de los genotipos se mantuvo a niveles similares en los tres años cubiertos por el estudio, esto puede sugerir que los genotipos evaluados en su conjunto presentan buena adaptabilidad a los diferentes ambientes de la región.

Los resultados del análisis de estabilidad y confiabilidad de la respuesta $\left(\mathrm{RN}_{\mathrm{i}}\right)$, para los 12 híbridos evaluados durante los últimos tres años en la región mesoamericana, permitieron identificar genotipos con rendimiento superior al testigo HB-83 (5,81 t/ha) y baja interacción con el ambiente, como CB-HS-11, TROP-E 103, HN-12 CMS933133 y H-59, estos genotipos presentaron puntuaciones AMMI cercanas a cero. No obstante, el análisis de confiabilidad permitió clasificarlos en tres categorías con respecto al testigo, los genotipos CMS 933133, XM-77088 y XM-7706K presentaron buena confiabilidad $\left(0,8 \geq \mathrm{RN}_{\mathrm{i}}<0,9\right)$ las probabilidades normalizadas de estos tres híbridos, significa que por lo menos en ocho de cada 10 localidades hay una respuesta superior de estos genotipos con respecto al testigo (Cuadro 4).

Entre tanto, los híbridos CB-HS-9, H-59, 30F94, CB-HS-11, TROP E 103 y HN-12 presentaron confiabilidad promedio $\left(0,6 \geq \mathrm{RN}_{\mathrm{i}}<0,8\right)$. Finalmente, los genotipos TROP E 101, HR-99 y HR-93 mostraron baja confiabilidad $\left(0,3 \geq \mathrm{RN}_{\mathrm{i}}<0,6\right)$. Los híbridos con niveles de confiabilidad por debajo de 0,60 y hasta 0,50 representan un riesgo relativamente similar para el productor con respecto al HB-83, es decir, que el productor puede sembrar cualquiera de estos genotipos con resultados muy similares al alcanzado con el testigo, siempre que otros componentes económicos permanezcan sin alteraciones como el costo de la semilla.
Cuadro 4. Rendimiento promedio, diferencias en rendimiento, desviación estándar y confiabilidad en 12 híbridos de maíz de grano blanco con respecto al testigo HB-83. PCCMCA 1998-2000.

\begin{tabular}{lccccc}
\hline Híbridos & $\begin{array}{c}\text { Rend. } \\
(\mathbf{t} / \mathbf{h a})\end{array}$ & $\begin{array}{c}\text { Dif. } \\
(\mathbf{t} / \mathbf{h a})\end{array}$ & $\begin{array}{c}\text { Desv. Est. } \\
(\mathbf{t} / \mathbf{h a})\end{array}$ & $\begin{array}{c}\text { Punt. } \\
\text { AMMI }\end{array}$ & $\begin{array}{c}\text { Prob. } \\
\text { RNi }>\mathbf{0}\end{array}$ \\
\hline CMS933133 & 6,89 & 1,08 & 0,974 & $-0,24$ & $0,86 \mathrm{a}$ \\
XM-7708 & 6,83 & 0,94 & 0,956 & 0,80 & $0,83 \mathrm{a}$ \\
XM-7706 & 6,77 & 0,87 & 0,957 & $-0,55$ & $0,82 \mathrm{a}$ \\
CB-HS-9 & 6,37 & 0,48 & 0,709 & $-0,50$ & $0,75 \mathrm{~b}$ \\
H-59 & 6,28 & 0,47 & 0,789 & $-0,22$ & $0,73 \mathrm{~b}$ \\
30F-94 & 6,24 & 0,43 & 0,867 & $-0,40$ & $0,69 \mathrm{~b}$ \\
CB-HS-11 & 6,27 & 0,37 & 0,834 & 0,01 & $0,67 \mathrm{~b}$ \\
Trop E 103 & 6,10 & 0,29 & 0,777 & $-0,15$ & $0,64 \mathrm{~b}$ \\
HN-12 & 6,18 & 0,17 & 0,577 & 0,14 & $0,61 \mathrm{~b}$ \\
Trop E 101 & 6,17 & 0,16 & 0,821 & $-0,59$ & $0,58 \mathrm{c}$ \\
HR-99 & 5,75 & $-0,15$ & 0,823 & 0,92 & $0,43 \mathrm{c}$ \\
HR-93 & 5,07 & $-0,74$ & 1,261 & $-0,40$ & $0,28 \mathrm{c}$ \\
HB-83 & 5,81 & & & $-0,05$ & \\
\hline
\end{tabular}

Nivel de confiabilidad: a Buena, b Promedio, c Baja.

Las magnitudes de las respuestas normalizadas (RNI) obtenidas en este estudio, coinciden con las obtenidas por Nuland y Eskridge (1992), Eskridge y Mumm (1992), Eskridge et al. (1993), Hodges et al. (1995), Córdova et al. (1993), Camargo et al (2001). En el estudio de Córdova et al. (1993), las ganancias en rendimiento sobre el híbrido H-5 fueron más impactantes al presentar genotipos con confiabilidad superior (H30 y HB-85). Entre mayor es la diferencia de rendimiento entre el genotipo evaluado y el testigo, más elevada es la confiabilidad.

De acuerdo con Lin et al. (1986), los análisis de estabilidad representan una medida relativa que permite hacer inferencia solamente en función del grupo fijo de genotipos evaluados. Sin embargo, Eskridge et al. (1993), indican que la confiabilidad permite hacer inferencias amplias en relación con la estabilidad, ya que, solamente depende de las diferencias en rendimiento del testigo común y de un genotipo específico y no depende de otros cultivares. Según Eskridge y Mumm (1992), Eskridge et al. (1993), la confiabilidad es una medida relacionada con otros parámetros de estabilidad como el coeficiente de regresión bi de Finlay y Wilkinson (1963), la desviación del cuadrado medio $\mathrm{S}^{2}$ de Eberhart y Russell (1966) y la varianza de estabilidad de Shukla (1972). Córdova et al. (1993), indica que la confiabilidad es una medida que evalúa las ganancias en rendimiento con respecto al testigo, así como, la variabilidad de la respuesta en los diferentes ambientes, tal como se aprecia en la 
Figura 1, donde se presenta la relación observada entre la diferencia con respecto al HB-83 y la confiabilidad de la respuesta de los híbridos evaluados. Se verifica que entre mayor es la diferencia de rendimiento, mayor es la confiabilidad estimada. Por otro lado, la variación en confiabilidad para los genotipos dentro de un diferencial de rendimiento dado, se debe fundamentalmente a las diferentes magnitudes de las desviaciones estándares a través de ambientes (Figura 1).

Las curvas de dispersión y regresión lineal de las diferencias de rendimiento con respecto al testigo en función de la probabilidad normalizada de los tres cultivares con mayor confiabilidad, se presenta en la Figura 2. En ésta se aprecia como los tres cultivares presentan patrones muy similares de respuesta en comparación con el testigo. También se encontró, que los coeficientes de las ecuaciones de las regresiones son muy similares. Esta similitud sugiere que el híbrido HB-83 es un testigo que ha permitido discriminar eficientemente los cultivares en cuanto a producción de grano y estabilidad.
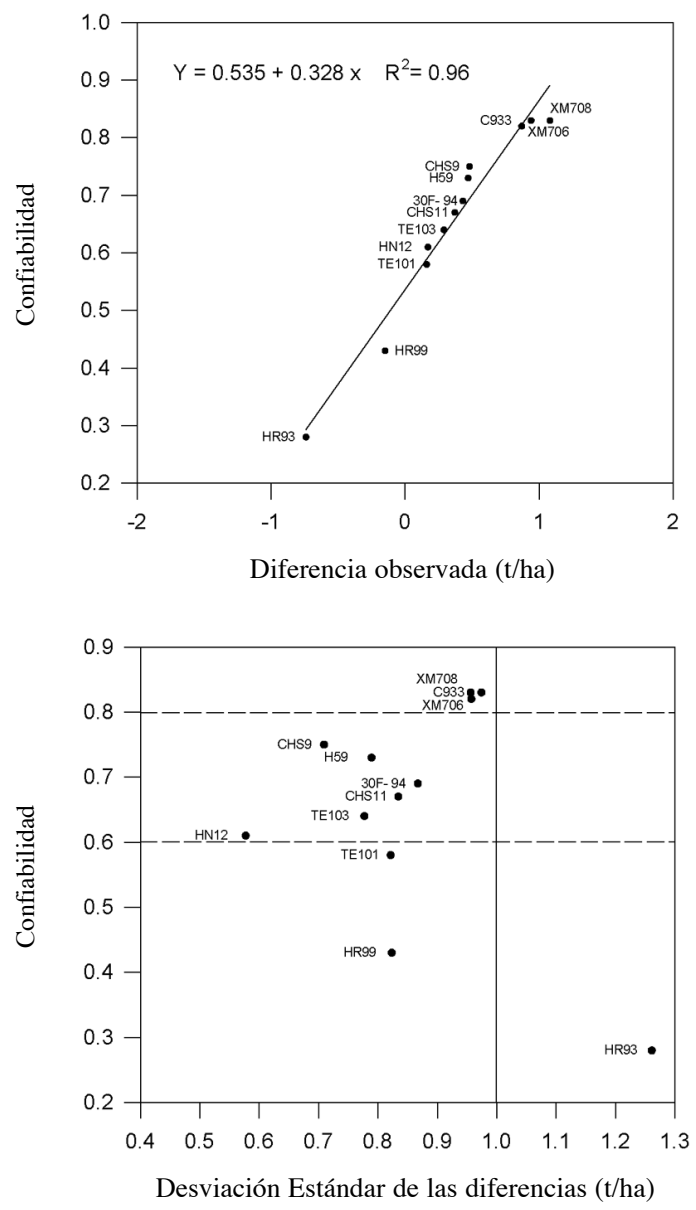

Figura 1. Relación de la confiabilidad con la magnitud de las diferencias en rendimiento, y la desviación estándar de 12 híbridos de maíz evaluados en el PCCMCA, 1998-2000.

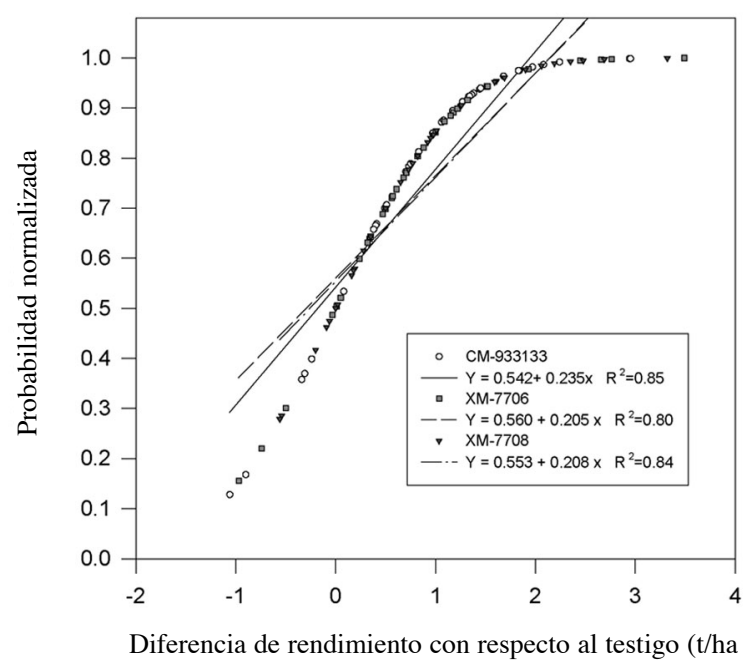

Figura 2. Relación de la probabilidad normalizada con las diferencias de rendimiento de tres híbridos de maíz evaluados en el PCCMCA, 1998-2002.

Como la confiabilidad normalizada permite la comparación de diferentes cultivares con un testigo común, la selección del testigo adecuado tiene un impacto importante en la estimativa de la confiabilidad, y en consecuencia sobre las conclusiones y decisiones a tomar (Eskridge y Mumm, 1992 y Eskridge et al. 1993). Por otro lado, Córdova et al. (1993), conceptúa que el testigo regional debe proveer tanto al mejorador como al productor un término de referencia común para comparar nuevos genotipos; por lo tanto, se requiere que su comportamiento ante todo sea estable y que tenga un nivel adecuado de producción.

El testigo regional HB-83, ha demostrado ser un genotipo excepcional puesto que combina altos rendimientos y baja interacción; estas características favorables contribuyen para que las respuestas normalizadas promedios de los nuevos cultivares no muestren ganancias espectaculares en rendimiento. Estimativas de las respuestas normalizadas fluctuando de buena a superior, implican incrementos en rendimiento de $20 \%$ sobre el HB-83 (5,81 t/ha).

La integración de los dos análisis presentados el de estabilidad AMMI y la confiabilidad normalizada, ayudan a aumentar la certeza del investigador y el productor en el proceso de decisión sobre la selección y/o liberación a nivel comercial de un genotipo en particular o bien identificar genotipos con características adecuadas para testigo y puede ser una manera indirecta de medir el progreso genético para rendimiento en función de un testigo regional. 


\section{CONCLUSIONES}

Se identificaron genotipos que combinan baja interacción con el ambiente y una respuesta normalizada de buena a promedio (CMS 933133, H-59, CBHS-11 y TROP E-103).

Por su comportamiento el híbrido CMS 933133, reúne los requisitos para ser utilizado como testigo regional.

El HB-83 continúa siendo un testigo regional vigente y competitivo por su alta producción y buena estabilidad a través de ambientes y años contrastantes.

Las metodologías evaluadas son útiles para identificar genotipos superiores, para su liberación o como testigos competitivos.

\section{LITERATURA CITADA}

CAMARGO, I.; GORDÓN, R.; FRANCO, J.; GONZÁLEZ, A.; QUIRÓS, E.; FIGUEROA, A. 2001. Confiabilidad de nuevos híbridos de maíz, en Panamá. Agronomía Mesoamericana 13(1): 07-11.

CÓRDOVA, H.S.; BARRETO, H.J.; CROSSA, J. 1993 Impacto del desarrollo de híbridos de maíz en Centro América: Confiabilidad de las ganancias en rendimiento sobre el genotipo $\mathrm{H} 5$ y consideraciones para selección de testigos regionales. In: Síntesis de resultados experimentales 1992. CIMMYT - PRM. Guatemala 1993. Programa Regional de Maíz para Centro América y el Caribe (PRM) 4: 3-10.

EBERHART, S; RUSSEL, W.A. 1966. Stability parameters for comparing varieties. Crop Sci 6:36-40.

ESKRIDGE, K.M. 1997 Evaluation of corn hybrids using the probability of outperforming a check based on strip-test data. Journal of agricultural, biological and environmental statistics 2(3): 245-254.

ESKRIDGE, K.M.; MUMM, V. 1992. Choosing plant cultivars based on the probability of outperforming a check. Theor. Appl. Genet. 84:494-500.

ESKRIDGE, K.M.; SMITH, O.S.; BYRNE, P.F. 1993. Comparing test cultivars using reliability functions of test- check differences from on-farm trials. Theor. Appl. Genet. 87:60-64.

FUENTES, M.; QUEMÉ, W. 1999. Evaluación de híbridos de maíz de grano amarillo y blanco en diferentes ambientes de Centroamérica, El Caribe, Colombia y Venezuela. Informe Ensayo Regional de Maíz PCCMCA,1998. ICTAPRM. Guatemala, C.A. 14 p.

FUENTES, M.; QUEMÉ, W. 2000. Evaluación de híbridos de maíz de grano amarillo y blanco en diferentes ambientes de Latinoamérica. Informe Ensayo Regional de Maíz PCCMCA, 1999. 14 p.

FUENTES, M.; QUEMÉ, W. 2001. Evaluación de híbridos de maíz de grano amarillo y blanco en diferentes ambientes de Centroamérica, México y El Caribe. Informe Ensayo Regional de Maíz PCCMCA,2000. ICTA- PRM. Guatemala, C.A. 18 p.

FINLAY, K.W.; WILKINSON, G.N. 1963. The analysis of adaptation in a plant-breeding programme. Aust J Agric Res 14:742-754.

Hill R., J.L. Rosenberger 1985. Methods for combining data from germplasm evaluation trials. Crop Sci. 25:467-470.

HODGES, L.; SANDERS, D.C.; PERRY, K. B. 1995. Adaptability and reliability of yield for four bell pepper cultivars across three southeastern states. Hort Sciences 30(6):1205-1210.

JONES, T.A. 1988. A probability method for comparing varieties against checks. Crop Sci. 28:907-912.

LIN, C.S.; BINNS, M.R. 1994. Concepts and methods for analyzing regional trial data for cultivar and location selection. Plant Breeding Reviews 12:271-297.

LIN C.S., BINNS., M.R.; LEFKOVITCH, L.P. 1986. Stability analysis: Where do we stand? Crop Sci. 26:894-900.

NULAND, D.S.; ESKRIDGE, K.M. 1992. Probability of outperforming a check. In: H.F. Schwartz (ed.). Proceedings, 35 th Bean Improvement Cooperative Meetings. Colorado State Univ. For. Collins. CO. p. 17-20.

ZOBEL, R.W.; MADISON, J.W.; GAUCH, H.G. JR. 1988. Statistical Analysis of a Yield Trial. Agron. J. 80:388393. 